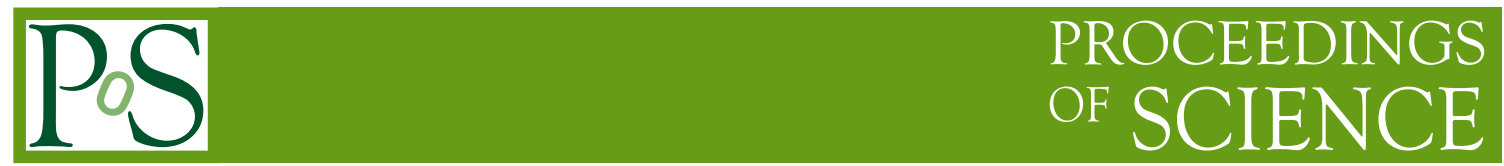

\title{
Overlap of di-neutron cluster state and shell model state
}

\author{
Makoto Ito, ${ }^{a, *}$ Riu Nakamoto, ${ }^{a}$ Eiju Ueda ${ }^{a}$ and Noritaka Shimizu ${ }^{b}$ \\ ${ }^{a}$ Department of Pure and Applied Physics, Kansai University, \\ 3-3-35 Yamatecho, Suita, Osaka, Japan \\ ${ }^{b}$ Center for Nuclear Study, The University of Tokyo, \\ 7-3-1 Hongo, BUnkyo-ku, Tokyo, Japan
}

E-mail: itomk@kansai-u.ac.jp, k298755@kansai-u.ac.jp

We formulate the computational method to calculate the overlap integral of the shell-model and cluster-model configurations. The framework is applied to the systems of the core plus two neutrons $(n)$, and the magnitude of the overlap of the shell model configuration (core $+n+n)$ and the di-neutron cluster one (core $+2 n)$ are explored. The overlap integrals are systematically calculated in the systems with the $j j$-closed cores, and we have found that the enhancement due to the occupation of the $s$ or $p$ orbit occurs in the systematic calculations.

\footnotetext{
*** Particles and Nuclei International Conference - PANIC2021 ***

*** 5 - 10 September, $2021 * * *$

*** Online ***
}

${ }^{*}$ Speaker 


\section{Introduction}

Nuclear shell model is a standard model to explain ground and low-lying states in nuclear systems [1]. In the ground state of a nucleus, all nucleons perform the independent particle motion inside of the self-consistent mean-field, and the several low-excited states can be described by the excitation of the independent particle motion [1]. On the contrary, in the lighter mass systems, there appears the clustering phenomena, in which the several nucleons are spatially localized and form subunits called clusters. A typical and well known example of such a cluster is the $\alpha$ cluster [2-4], which is a quartet of two proton plus two neutrons. In the ground state, the $\alpha$ cluster and the residual nucleus are merged, which finally goes to the shell model structure, but the $\alpha$ clustering is much developed in the excited states [2-4].

The independent particle configuration in the shell model seems to contradict the cluster configuration with a spatial localization of the several nucleons but these two configurations are non-orthogonal, and hence the amplitude of the cluster formation is non-zero even if the pure shell model structure is realized, in which the individual nucleons completely perform the independent particle motions. The relation of the shell model and the $\alpha$-cluster model configurations is deeply investigated in the lighter mass systems, especially in the mass region of $A \sim 20$ [5], but the relation still remains unclear in the heavier mass region.

The evaluation of the non-orthogonal amplitude of the shell and cluster models is important to clarify the relation of these two configurations, which seem to describe the different particle motions intuitively. In this article, we formulate a new method to calculate the overlap integral of the wave functions in the shell and cluster models by employing the Fourier transformation. The new framework is applied to the core + two neutrons systems, which have recently been discussed in connection to di-neutron correlations [6-9]. We investigate the basic feature in the overlap integrals of the naive shell model configuration and the di-neutron cluster one. All the results presented in this article are compressed from Ref. [10].

\section{Theoretical framework}

The shell model wave function $\Psi_{s}$ for two neutrons around the heavy core is constructed from the direct product of the single particle orbits, which is written in a symbolic form,

$$
\Psi_{s}=N \mathcal{A}\left\{\phi_{a}\left(\mathbf{r}_{1}\right) \phi_{b}\left(\mathbf{r}_{2}\right)\right\} .
$$

Here $\phi_{a}\left(\mathbf{r}_{i}\right)$ shows the single particle wave function for the $i$-th valence neutron. The subscripts $a$ and $b$ in $\phi$ are the abbreviation of a set of the $i$-th single particle orbit, such as $a \equiv\left(n, l, j, j_{z}\right)$ with the radial node $n$, the orbital spin $l$, the total spin $j$ and its third component $j_{z}$. The vector $\mathbf{r}_{i}$ contains a set of the coordinates for the single particle orbit: the position vector and the spin coordinates. Both of the single particle orbits are orthogonal to the orbits contained in the core nucleus, which is not considered explicitly in the present calculation. In Eq. (1), $\mathcal{A}$ and $N$ mean the anti-symmetrization operator for all nucleons and the respective normalization constant, respectively.

The wave function of the di-neutron $(2 n)$ cluster model is

$$
\Psi_{c}=N \mathcal{A}\left\{\chi_{L M}(\mathbf{R}) \varphi(\rho, \zeta)\right\}
$$


where $N, \mathcal{A}$ are the same symbols as those in Eq. (1): the normalization constant and the antisymmetrizer, respectively. $\chi_{L M}(\mathbf{R})$ denotes the wave function for the core $-2 n$ relative motion with the orbital spin $L$ and its third component $M$, which is a function of the core $-2 n$ relative coordinate, $\mathbf{R}$. Here we assume the $L=M=0$ state for simplicity.

In Eq. (2), $\varphi(\rho, \zeta)$ shows the internal wave function of the $2 n$ cluster with the spin-less neutron pair $(S=0)$. The coordinate $\rho$ denotes the $n-n$ relative coordinate, while $\zeta$ represents the spin coordinate. Here the spatial part in $\varphi(\rho, \zeta)$ is set to the simple Gaussian function corresponding to the $(0 s)^{2}$ configuration in the harmonic oscillator potential with the width parameter $v$.

We calculate the shell-cluster overlap, $\left\langle\Psi_{s} \mid \Psi_{c}\right\rangle$, by combing the Gaussian expansions for the radial wave functions, which appears in Eqs. (1) and (2), and the Fourier transformation. The explicit expression of overlap becomes quite simple, and it is given in Ref. [10].

\section{Results}

We have calculated the overlap integral of the shell model configuration (core $+n+n)$ and the di-neutron cluster configuration (core $+2 n$ ) in the various systems with the $j j$-closed cores, such as ${ }^{16} \mathrm{O},{ }^{28} \mathrm{Si},{ }^{32} \mathrm{~S},{ }^{40} \mathrm{Ca}$ and ${ }^{56} \mathrm{Ni}$. In Fig. 1 , we show the results of the systematic calculations of the shell-cluster overlap integrals. In this calculation, the harmonic oscillator (HO) wave function is applied to all of the radial wave functions The internal width parameter of the $2 n$ cluster $(v)$ is fixed so as to reproduce the root-mean-squared radius of a deuteron $(\sim 2.1 \mathrm{fm})$.

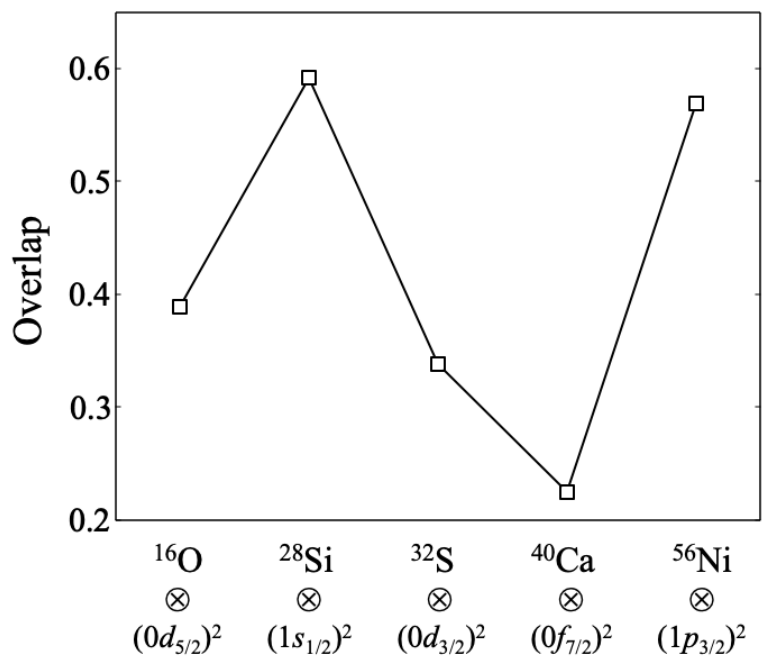

Figure 1: Systematic calculations of shell-cluster overlap. In the abscissa, the combination of the core and the shell configuration of two neutrons is shown.

In the calculation shown in Fig. 1, the lowest shell model configuration is assumued for the valence two neutrons, which couples to the spin zero pair, such as $\mathbf{j}_{1} \otimes \mathbf{j}_{2}=\mathbf{J}=0$. In Fig. 1, the prominent enhancements can be seen at the core of ${ }^{28} \mathrm{Si}$ and ${ }^{56} \mathrm{Ni}$, which corresponds to the two neutrons configurations of $\left(1 s_{1 / 2}\right)^{2}$ and $\left(1 p_{3 / 2}\right)^{2}$, respectively. This result means that the shellcluster overlap is increased if the two neutrons occupy the shell model orbit having the lower orbital spins, such as $l=0$ and 1 . 


\section{Summary}

In summary, we have formulated the computational technique to calculate the shell-cluster overlap integral. The formulation is achieved by combing the Gaussian expansion of the radial wave function and the Fourier transformation. Although we do not show the final expression of the shell-cluster overlap, it becomes quite simple from, which is given by the direct product of the kinetic part and the dynamical part. The former and latter parts are determined by the angular momentum algebra and the radial wave functions, respectively [10].

Our formula has been applied to the systems of the core plus valence two neutrons, and the shell-cluster overlap is evaluated in the systems with the $j j$-closed cores. In the systematic calculations, we have found the enhancement in the overlap when two neutrons occupy the shell model orbits having the lower orbital angular momentum, such as the $s$ and $p$ wave orbits.

We have also considered the effect of the configuration interaction (CI) in the calculation of the overlap integrals by employing the computational code of K-shell [11]. The CI effect, which gives rise to the mixture of the component of the higher orbital spin, increases the magnitude of the overlap integral by a factor of $1.3 \sim 2$ but its amount is almost constant, and hence the original peak structure appearing at the $s$ and $p$ orbits survived after the CI effect is switched on.

It is very interesting to study the overlap integral in the systems of the core plus four nucleons. It is possible to extend our formulation to the core $+4 N$ systems, which correspond to the $\alpha$ cluster systems, in a straight forward manner. Analysis of the overlap in the four nucleons systems is now under progress.

\section{References}

[1] P. Ring and P. Schuck, 2004 Nuclear Many-Body Problem1st edn (Berlin: Springer), and references therein.

[2] K. Ikeda et al., Prog. Theor. Phys. Suppl. 68, 1 (1986).

[3] H. Horiuchi et al., Suppl. Prog. Theor. Phys. 192, 1 (2012), and references therein.

[4] H. Horiuchi et al., Prog. Theor. Phys. Suppl. 62, 1 (1980).

[5] T. Yamada, Y. Funaki, H. Horiuchi, K. Ikeda, and A. Tohsaki, Prog. Theor. Phys. 120, 1139 (2008), and references therein.

[6] K. J. Cook, et al., Phys. Rev. Lett. 124, 212503 (2020).

[7] K. Kubota et al., Phys. Rev. Lett. 125, 212501 (2020).

[8] K. Hagino and H. Sagawa, Phys. Rev. C 72, 044321 (2005).

[9] Y. Kikuchi, K. Ogata, Y. Kubota, M. Sasano, and T. Uesaka, Prog. Theor. Exp. Phys. 2016, $103 \mathrm{D} 03$.

[10] R. Nakamoto, M. Ito, E. Ueda and N. Shimizu, Submitted to Prog. Theor. Phys. (2021).

[11] N. Shimizu, T. Mizusaki, Y. Utsuno and Y. Tsunoda, Comp. Phyis. Comm. 244, 372-384 (2019). 